\title{
State equations in the mathematical model of dynamic behaviour of multihull floating unit
}

\author{
Agnieszka Królicka, M. Sc. \\ Gdansk University of Technology
}

\begin{abstract}

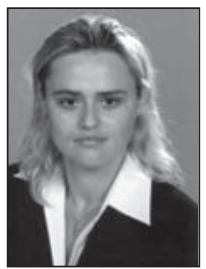

This paper concerns dynamic behaviour of multihull floating unit of catamaran type exposed to excitations due to irregular sea waves. Dynamic analysis of multihull floating unit necessitates, in its initial stage, to determine physical model of the unit and next to assume an identified mathematical model. Correctly elaborated physical models should contain information on the basis of which a mathematical model could be built. Mathematical models describe mutual relations between crucial quantities which characterize a given system in time domain. The dynamic analysis of multihull unit was performed under assumption that the unit's model has been linear and exposed to action of irregular sea waves. Mathematical model of such dynamic system is represented by state equations. The formulated equations take into account encounter of head wave which generates symmetrical motions of the unit, i.e. surge, heave and pitch. For solving the equations the following three wave spectra were taken into consideration:
\end{abstract}

- ISSC (International Ship Structures Congress) spectrum

- Pierson-Moskowitz spectrum

- Paszkiewicz spectrum.

Keywords: dynamics of multihull floating unit, sea waves, stochastical processes, wave spectra

\section{INTRODUCTION}

Motion of a dynamic system can be generated by diferent external or internal factors.At mathematical modelling external excitation factors of the most significant effect on the system, are selected. Such external factors are usually called excitations. Response of the system to given excitations is mathematically characterized by a definite transformation called operator of a system. For a broad class of dynamic systems the relation between excitations and response is characterized by differential equations of motion. The equations can be linear or non-linear of constant or varying coefficients, ordinary or dif ferential, deterministic or stochastic ones. The mathematical models used for practical applications almost always necessitate to simplify the equations which form a given model. It amounts mainly to lowering their order by forming partial models and time - local ones with an assumed practical accuracyDynamic mechanical systems which represent floating objects are tightly associated with stochastic processes. State variables and input parameters of the models are of probabilistic character. Mathematical models of such systems are represented by sets of stochastic dif ferential equations, and form sets of Itô Itequations. Multihull units such as catamarans and trimarans belong to complex, highly non-linear dynamic systems. If dynamic system model is of floating unit's linear system then the equations:

$$
\sum_{i, j=1}^{6} I_{i j} \ddot{\eta}_{i}+B_{i j} \dot{\eta}_{i}+C_{i j} \eta_{i}=F_{i}(t)
$$

where:

$\mathrm{I}=\mathrm{M}+\mathrm{A}-$ inertia matrix

M - elements of matrix of generalized masses of structures

A - elements of matrix of generalized hydrodynamic added masses

B - hydrodynamic damping matrix

C - hydrostatic stiffness matrix

$\eta \quad-$ generalized displacement vector

$\mathrm{F}(\mathrm{t}) \quad-$ vector of exciting forces and moments

can be analyzed as a set of two mutually non-coupled groups of mutually coupled equations. It is assumed that the coupling takes place by linear and non-linear damping coefficients and hydrostatic stiffness coefficients.

In these considerations the examined object is taken as a linear dynamic system of six degrees of freedom.

Among them are the following:

- translational oscillations:

a) surging $-\eta_{1}$

b) swaying $-\eta_{2}$

c) heaving $-\eta_{3}$

- angular oscillations:

d) rolling $-\eta_{4}$

e) pitching $-\eta_{5}$

f) yawing $-\eta_{6}$

The catamaran-fixed coordinate frame is shown in Fig. 1. 


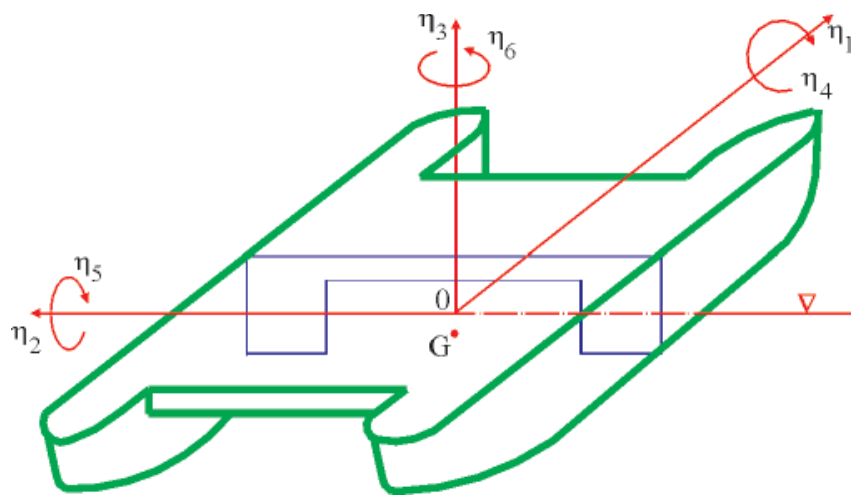

Fig. 1. Schematic diagram of physical model of catamaran

Local motions of the unit constitute its response to seawave-induced excitations. To the first group belong the equations which describe the symmetrical motions: $\eta_{1}, \eta_{3}, \eta_{5}$. The second group is formed by the equations which describe the anti-symmetrical motions: $\eta_{2}, \eta_{4}, \eta_{6}$. Constructional reasons (symmetry) of multihull floating units make it possible to analyze group-coupled motions of the object and in consequence to limit number of state variables which appear in the equations.

\section{SET OF STATE EQUATIONS}

State equations are one of the possible ways for representing mathematical model of a dynamic system. An alternative way to describe a dynamic system is transmittance in which the initial state is assumed equal to zero.

The operator transmittance, otherwise called the transfer function $\mathrm{G}(\mathrm{s})$, is ratio of output signal Laplace transform and input signal Laplace transform of the system in zero initial conditions. The transmittance describes general features of a stationary linear system of one input and one output, independing on a kind of excitation. For the systems described by linear dif ferential equations of constant coefficients the transmittance is rational function of the complex variable s, which can be represented by the quotient of two polynomials (2).

To determine the set of state equations the following assumptions have been applied:

1) resultant motion of the object on an irregular wave is formed by superposing its motions in regular waves

2) only encounter of the head wave which generates only the motions: surging $\eta_{1}$, heaving $\eta_{3}$ and pitching $\eta_{5}$, is taken ito consideration

3 ) it is assumed that the response of the object, in the form of the generalized wave-generated forces $F_{i}(i=1,3,5)$, to excitation due to the wave of the height $\xi^{1}(\mathrm{t})$, can be approximated by means of a system whose transmittance is of the form:

$$
\frac{F(s)}{\xi(s)}=\frac{b_{0} s^{2}+b_{1} s+b_{2}}{s^{2}+a_{1} s+a_{2}}
$$

where:

$\mathrm{F}\left(\mathrm{F}_{1}, \mathrm{~F}_{3}, \mathrm{~F}_{5}\right)$

$\mathrm{b}_{0}\left(\mathrm{~b}_{01}, \mathrm{~b}_{03}, \mathrm{~b}_{05}\right), \mathrm{b}_{1}\left(\mathrm{~b}_{11}, \mathrm{~b}_{13}, \mathrm{~b}_{15}\right), \mathrm{b}_{2}\left(\mathrm{~b}_{21}, \mathrm{~b}_{23}, \mathrm{~b}_{25}\right)$

$a_{1}\left(a_{11}, a_{13}, a_{15}\right), a_{2}\left(a_{21}, a_{23}, a_{25}\right)$.

The relation (2) can be written by using the following set of state equations:

$$
\begin{gathered}
\mathrm{f}_{1}=\mathrm{F}-\mathrm{h}_{0} \xi \\
\mathrm{f}_{2}=\dot{\mathrm{F}}-\mathrm{h}_{0} \dot{\xi}-\mathrm{h}_{\mathrm{1}}
\end{gathered}
$$

$$
\left.\begin{array}{l}
\dot{\mathrm{f}}_{1}=\mathrm{f}_{2}+\mathrm{h}_{1} \xi \\
\dot{\mathrm{f}}_{2}=-\mathrm{a}_{2} \mathrm{f}_{1}-\mathrm{a}_{1} \mathrm{f}_{2}+\mathrm{h}_{2} \xi
\end{array}\right\}
$$

where:

$\mathrm{h}_{0}, \mathrm{~h}_{1}, \mathrm{~h}_{2}-$ constants defined by the coefficients of the equation (2)

$\mathrm{f}_{1}\left(\mathrm{f}_{11}, \mathrm{f}_{13}, \mathrm{f}_{15}\right), \mathrm{f}_{2}\left(\mathrm{f}_{21}, \mathrm{f}_{23}, \mathrm{f}_{25}\right)$

$\mathrm{h}_{0}\left(\mathrm{~h}_{01}, \mathrm{~h}_{03}, \mathrm{~h}_{05}\right), \mathrm{h}_{1}\left(\mathrm{~h}_{11}, \mathrm{~h}_{13}, \mathrm{~h}_{15}\right), \mathrm{h}_{2}\left(\mathrm{~h}_{21}, \mathrm{~h}_{23}, \mathrm{~h}_{25}\right)$.

To obtain random process of the irregular wave height $\xi(\mathrm{t})$ the well-known energy (wave) spectra of the wave which encounters the object, can be used:

$\phi_{\xi \xi}(\omega)=\left\{\begin{array}{l}\text { e.g. Pierson }- \text { Moskowitz spectrum } \\ \text { or ISSC spectrum }\end{array}\right.$

The selected spectrum is approximated by means of the spectral density function in the form:

$$
g(\omega)=\frac{C \omega^{2}}{\omega^{4}-2 v \omega_{0}^{2} \omega^{2}+\omega_{0}^{4}}
$$

Then, the shape filter is introduced by making use of the following assumptions:

1. In both the spectra, i.e.(5) and (6), their maxima appear at the same frequency and are of the same value

2. $\int_{0}^{\infty} \phi_{\xi \xi}(\omega) \mathrm{d} \omega=\int_{0}^{\infty} \mathrm{g}(\omega) \mathrm{d} \omega$

3. The wave height processes are generated by the transfer function G(s) which constitutes the so-called shape filter if ,white noise" is at input.

The transfer function G(s) is given by the relation:

$$
\mathrm{G}(\mathrm{s})=\frac{\mathrm{a}_{0} \mathrm{~s}}{\mathrm{~s}^{2}+\mathrm{a}_{1} \mathrm{~s}+\mathrm{a}_{2}}
$$

The relation (7) corresponds to the following set of state equations:

$$
\left.\begin{array}{c}
\mathrm{g}_{1}=\xi \\
\mathrm{g}_{2}=\dot{\xi}-\mathrm{a}_{0} \mathrm{~W}
\end{array}\right\}
$$

where:

W - „white noise"

$\xi(\mathrm{t})$ - wave height process.

The above given matrix ( $\mathrm{T}$ ab. 1) can be written in the general form of Itô equation:

$$
\dot{\mathrm{X}}=\widetilde{\mathrm{A} X}+\widetilde{\mathrm{B} W}
$$

From the matrix the following is obtained:

$$
\left.\begin{array}{l}
\dot{f}_{11}=f_{21} \\
\dot{f}_{21}=-a_{21} f_{11}-a_{11} f_{21} \\
\dot{f}_{13}=f_{23} \\
\dot{f}_{23}=-a_{23} f_{13}-a_{13} f_{23} \\
\dot{f}_{15}=f_{25} \\
\dot{f}_{25}=-a_{25} f_{15}-a_{15} f_{25} \\
\dot{g}_{1}=g_{2}+a_{0} W \\
\dot{g}_{2}=-a_{2} g_{1}-a_{1} g_{2}-a_{0} a_{1} W
\end{array}\right\}
$$


Tab. 1. Matrix of state equations

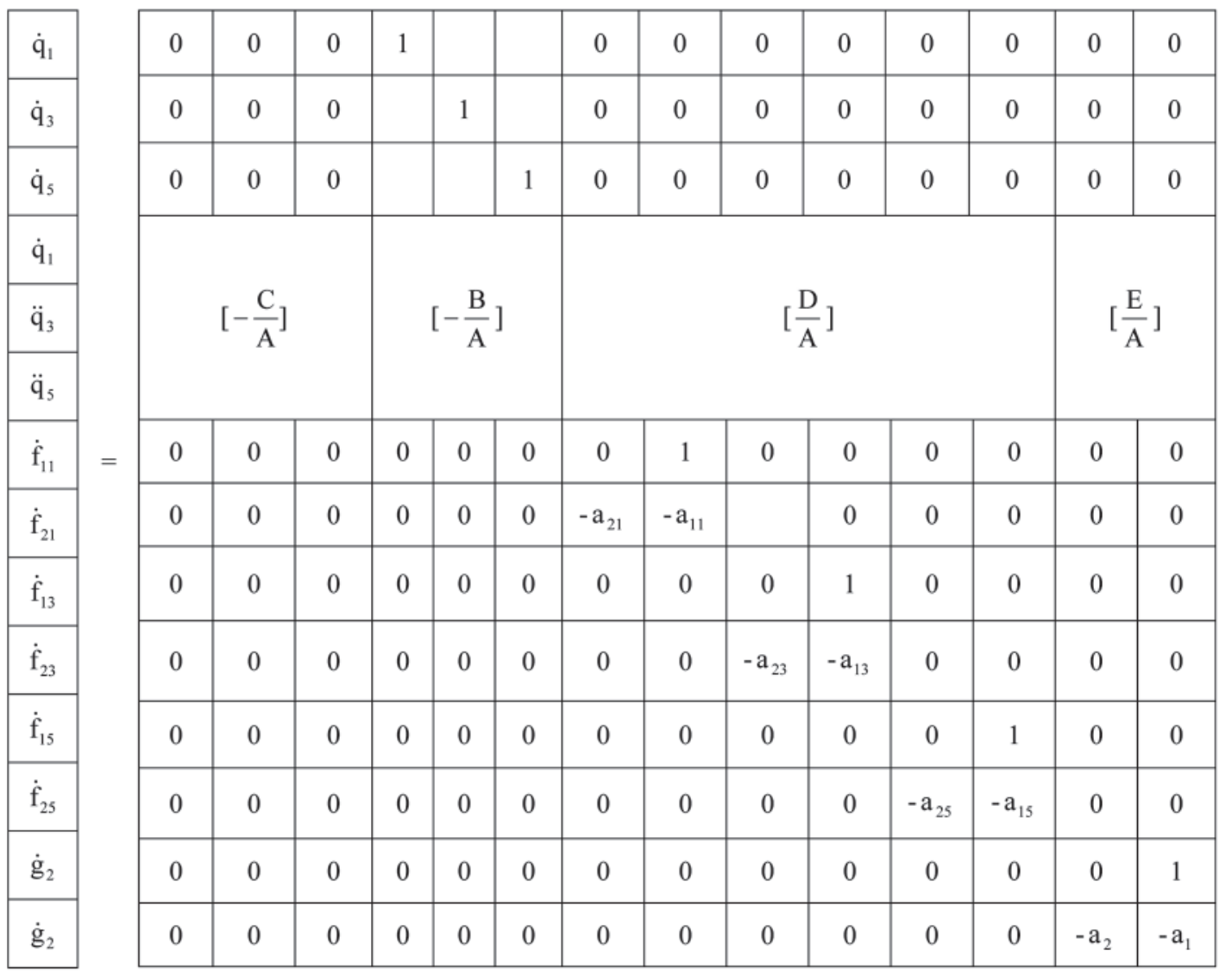

\begin{tabular}{|c|}
\hline $\mathrm{q}_{1}$ \\
\hline $\mathrm{q}_{3}$ \\
\hline $\mathrm{q}_{5}$ \\
\hline$\dot{\mathrm{q}}_{1}$ \\
\hline$\dot{\mathrm{q}}_{3}$ \\
\hline$\dot{\mathrm{q}}_{5}$ \\
\hline $\mathrm{f}_{11}$ \\
\hline $\mathrm{f}_{21}$ \\
\hline $\mathrm{f}_{13}$ \\
\hline $\mathrm{f}_{23}$ \\
\hline $\mathrm{f}_{15}$ \\
\hline $\mathrm{f}_{25}$ \\
\hline $\mathrm{g}_{1}$ \\
\hline $\mathrm{g}_{2}$ \\
\hline 0 \\
\hline 0 \\
\hline 0 \\
\hline 0 \\
\hline 0 \\
\hline $\mathrm{a}_{0}$ \\
\hline$-\mathrm{a}_{0} \mathrm{a}_{1}$ \\
\hline
\end{tabular}

where:

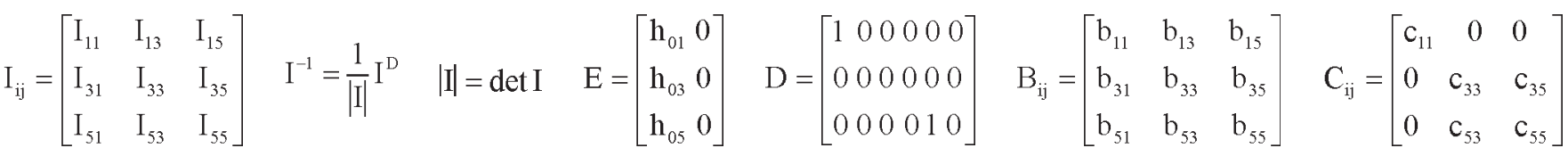

The parameters $\ddot{\mathrm{q}}_{1}, \ddot{\mathrm{q}}_{2}, \ddot{\mathrm{q}}_{3}$ can be determined after finding and solving the particular coefficients of damping, hydrostatic stiffness as well as inertia ones.

\section{EXAMPLE SOLUTIONS FOR THE SELECTED WAVE SPECTRA}

\section{ISSC wave spectrum}

$$
\phi(\omega)=\frac{\mathrm{A}}{\omega^{5}} \exp \left(-\frac{\mathrm{B}}{\omega^{4}}\right)
$$

where:

$$
\left.\begin{array}{l}
\mathrm{B}=691\left(\frac{2 \pi}{\omega_{1}}\right)^{-4} \\
A=173 \mathrm{~h}_{1 / 3}^{2}\left(\frac{2 \pi}{\omega_{1}}\right)^{-4}
\end{array}\right\}
$$

The wave spectrum is approximated by means of the spectral density function of the following form:

$$
g(\omega)=\frac{C \omega^{2}}{\omega^{4}-2 v \omega_{0}^{2} \omega^{2}+\omega_{0}^{4}}
$$

Let it be:

$$
\left.\begin{array}{rl}
2 \mathrm{v} \omega_{0}^{2} & \equiv \mathrm{D} \\
\omega_{0}^{4} & \equiv \mathrm{E}
\end{array}\right\}
$$

Now $\omega$ - value at which the extremum appears, is determined, i.e.:

$$
\begin{aligned}
& g(\omega)=\frac{\mathrm{C} \omega^{2}}{\omega^{4}-\mathrm{D} \omega^{2}+\mathrm{E}} \\
& \frac{\partial}{\partial \omega}[\phi(\omega)]=\frac{\partial}{\partial \omega}[\mathrm{g}(\omega)]
\end{aligned}
$$

From the condition that extremum is present in the same point the following is obtained:

i.e. the first equation:

$$
\left(\frac{4 B}{5}\right)^{\frac{1}{4}}=E^{\frac{1}{4}} \text { so } E=\frac{4}{5} B
$$

From the second assumption that:

$$
\begin{aligned}
& \phi(\omega)=g(\omega) \\
& \left|\omega=\left(\frac{4}{5} B\right)^{\frac{1}{4}}\right| \omega=E^{\frac{1}{4}}
\end{aligned}
$$

the following is obtained:

i.e. the second equation:

$$
\frac{5 \mathrm{~A} \sqrt[4]{5}}{4 \mathrm{~B} \sqrt[4]{4 \mathrm{~B}}} \mathrm{e}^{\frac{4}{5}}=\frac{C}{2 \sqrt{\mathrm{E}}-\mathrm{D}}
$$


and the third equation

$$
\int_{0}^{\infty} \frac{\mathrm{C} \omega^{2}}{\omega^{4}-\mathrm{D} \omega^{2}+\mathrm{E}} \mathrm{d} \omega=\int_{0}^{\infty} \frac{\mathrm{A}}{\omega^{5}} \exp \left(-\frac{\mathrm{B}}{\omega^{4}}\right) \mathrm{d} \omega
$$

From the right-hand side of the Eq. (20):

$$
\int_{0}^{\infty} \frac{\mathrm{A}}{\omega^{5}} \exp \left(-\frac{\mathrm{B}}{\omega^{4}}\right) \mathrm{d} \omega=\mathrm{A} \int_{0}^{\infty} \omega^{-5} \exp \left(\frac{-\mathrm{B}}{\omega^{4}}\right) \mathrm{d} \omega
$$

by substituting:

$$
\begin{gathered}
\omega^{-4} \equiv \mathrm{t} \\
-4 \omega^{-5} \mathrm{~d} \omega \equiv \mathrm{dt} \Rightarrow \mathrm{d} \omega=-\frac{1}{4} \omega^{5} \mathrm{dt}
\end{gathered}
$$

the following is achieved:

$$
\begin{gathered}
\mathrm{A} \int_{0}^{\infty} \omega^{-5} \exp (-\mathrm{B} \mathrm{t})\left[-\frac{1}{4} \omega^{5} \mathrm{dt}\right]= \\
=-\frac{\mathrm{A}}{4} \int_{0}^{\infty} \exp (-\mathrm{B} \mathrm{t}) \mathrm{dt}=\left.\frac{\mathrm{A}}{4 \mathrm{~B}} \mathrm{e}^{-\mathrm{Bt}}\right|_{0} ^{\infty}=-\frac{\mathrm{A}}{4 \mathrm{~B}}
\end{gathered}
$$

The left-hand side of Eq. (20) is of the form:

$$
\int_{0}^{\infty} \frac{C \omega^{2}}{c \omega^{4}-b \omega^{2}+a} d \omega
$$

in which „C" means ,white noise”, and the coefficients a, b, c are equal to:

$$
\left.\begin{array}{l}
\mathrm{b}=2 \mathrm{v} \omega_{0}^{2} \\
\mathrm{a}=\omega_{0}^{4} \\
\mathrm{c}=1
\end{array}\right\}
$$

After solving the above given integral the final solution is achieved as follows:

$$
\begin{gathered}
\int_{0}^{\infty} \frac{\mathrm{C} \omega^{2}}{\omega^{4}-\mathrm{D} \omega^{2}+\mathrm{E}} \mathrm{d} \omega= \\
2 \omega_{0}\left[\left(\mathrm{v}-{\left.\sqrt{\mathrm{v}^{2}-1}\right)^{\frac{1}{2}}+\left(\mathrm{v}+{\sqrt{\mathrm{v}^{2}-1}}^{\frac{1}{2}}\right]}^{-\mathrm{C} \pi}\right.\right.
\end{gathered}
$$

By comparison of the left hand side and right hand side (the third equation) the following is obtained:

$$
-\frac{A}{4 B}=\frac{-C \pi}{2 \omega_{0}\left[\left(v-\sqrt{v^{2}-1}\right)^{\frac{1}{2}}+\left(v+\sqrt{v^{2}-1}\right)^{\frac{1}{2}}\right]}
$$

This way the third, lacking equation for the unknowns C, $\mathrm{v}, \omega_{0}$, has been achieved.

The first assumption (Eq. (18)) yields the following:

$$
\mathrm{E} \equiv \mathrm{a}=\omega_{0}^{4}=\frac{4}{5} \mathrm{~B} \Rightarrow \omega_{0}^{2}= \pm \sqrt{\frac{4 \mathrm{~B}}{5}}
$$

The second equation (Eq. (19)) yields the relation:

$$
\frac{5 \mathrm{~A}}{4 \mathrm{~B}}\left(\frac{5}{4 \mathrm{~B}}\right)^{\frac{1}{4}} \mathrm{e}^{\frac{4}{5}}=\frac{\mathrm{C}}{2 \sqrt{\mathrm{E}}-\mathrm{D}}
$$

By introducing the following substitution:

$$
\left.\begin{array}{l}
\mathrm{D} \equiv \mathrm{b}=2 \mathrm{v} \omega_{0}^{2} \\
\mathrm{E} \equiv \omega_{0}^{4}
\end{array}\right\}
$$

the relation is achieved:

$$
\frac{5 \mathrm{~A}}{4 \mathrm{~B}}\left(\frac{5}{4 \mathrm{~B}}\right)^{\frac{1}{4}} \cdot \mathrm{e}^{\frac{4}{5}}=\frac{\mathrm{C}}{2 \omega_{0}^{2}(1-\mathrm{v})}
$$

The constants $\mathrm{A}$ and $\mathrm{B}$ are equal to:

$$
\left.\begin{array}{l}
B \equiv 691\left(\frac{2 \pi}{\omega_{1}}\right)^{-4} \\
A \equiv 173 h_{S}^{2}\left(\frac{2 \pi}{\omega_{1}}\right)^{-4}
\end{array}\right\}
$$

From Eq. (30) is obtained the quantity $\mathrm{C}$ expressed as follows:

$$
\mathrm{C}=\frac{2 \mathrm{~A}(1-\mathrm{v})}{\omega_{0}^{3} \cdot \mathrm{e}^{5 / 4}}
$$

From Eq. (26) is determined the quantity $\mathrm{C}$ equal to:

$$
\mathrm{C}=\frac{2 \mathrm{~A} \omega_{0}}{4 \pi \mathrm{B}}\left[\left(\mathrm{v}-\sqrt{\mathrm{v}^{2}-1}\right)^{\frac{1}{2}}+\left(\mathrm{v}+\sqrt{\mathrm{v}^{2}-1}\right)^{\frac{1}{2}}\right.
$$

Next the right hand sides of the above given equations are compared to each other to get the equation for determining the unknown ,v".

$$
\left.\begin{array}{l}
\mathrm{v}_{1}=1+\frac{1}{\mathrm{~L}^{2}}+\frac{\sqrt{4 \mathrm{~L}^{2}+1}}{\mathrm{~L}^{2}} \\
\mathrm{v}_{2}=1+\frac{1}{\mathrm{~L}^{2}}-\frac{\sqrt{4 \mathrm{~L}^{2}+1}}{\mathrm{~L}^{2}}
\end{array}\right\}
$$

Now the quantity $\mathrm{L}=4.500406826$ and its square $\mathrm{L}^{2}=20.2536616$ is determined.

Hence:

$$
\left.\begin{array}{l}
v_{1}=1.496512384 \\
v_{2}=0.602235192
\end{array}\right\}
$$

By substituting $\mathrm{v}_{1}$ the following is obtained:

$$
\begin{gathered}
\mathrm{a}_{2}=0.596 \omega_{1}^{2} \\
\mathrm{C} \cong-0.068635565 \mathrm{~h}_{\mathrm{S}}^{2} \cdot \omega_{1} \\
\mathrm{a}_{0}=\sqrt{\mathrm{C}} \cong 0.261983903 \mathrm{~h}_{\mathrm{s}} \sqrt{\omega_{1}} \\
\mathrm{a}_{1}=\omega_{0} \sqrt{2(1-\mathrm{v})}= \\
=0.772010362 \omega_{1} \cdot 0.996506281 \cong \\
\cong 0.769313175 \omega_{1}
\end{gathered}
$$

By substituting $\mathrm{v}_{2}$ the following is obtained:

$$
\left.\begin{array}{c}
\mathrm{C}=\frac{137.6266236}{2502.977614}=0.054985159 \mathrm{~h}_{\mathrm{S}}^{2} \cdot \omega_{1} \\
\mathrm{a}_{0}=\sqrt{\mathrm{C}} \cong 0.234489144 \mathrm{~h}_{\mathrm{S}} \sqrt{\omega_{1}} \\
\mathrm{a}_{1}=0.772010362 \omega_{1} \cdot 0.891924669 \cong \\
\cong 0.688575087 \omega_{1}
\end{array}\right\}
$$


2. Pierson-Moskowitz, wave spectrum

$$
\begin{gathered}
\left.\begin{array}{c}
\phi=\frac{\mathrm{A}}{\omega^{5}} \mathrm{e}\left(-\frac{\mathrm{B}}{\omega^{4}}\right) \\
\mathrm{T}_{1}=0.771 \mathrm{~T}_{0} \\
\mathrm{~T}_{2}=0.92 \mathrm{~T}_{1}
\end{array}\right\} \\
\mathrm{A}=\frac{1}{8 \pi^{2}}\left(\frac{2 \pi}{\mathrm{T}_{2}}\right)^{5}=\frac{1}{8 \pi^{2}}\left(\frac{2 \pi}{0.92 \mathrm{~T}_{1}}\right)^{5}= \\
=\frac{1}{8 \pi^{2}(0.92)^{5}}\left(\frac{2 \pi}{\mathrm{T}_{1}}\right)^{5}=188.17 \\
\mathrm{~B}=\frac{1}{\pi}\left(\frac{2 \pi}{\mathrm{T}_{1}}\right)^{4}=496.1
\end{gathered}
$$

On the assumption that: $\mathrm{L}=4.500406826$ and $\mathrm{v}_{1}=1.496512384, \mathrm{v}_{2}=0.602235192$, value of the constant $\mathrm{C}$ is calculated:

$$
\begin{gathered}
\mathrm{C}=\frac{188.1787 \mathrm{~h}_{\mathrm{S}}^{2} 0.71037 \omega_{1} \cdot 2.234507723}{2 \pi \cdot 496.1}= \\
=\mathrm{h}_{\mathrm{S}}^{2} \cdot \omega_{1} \cdot 0.095826987 \\
\mathrm{a}_{0}=\sqrt{\mathrm{C}}=0.309559344 \cdot \mathrm{h}_{\mathrm{S}} \cdot \sqrt{\omega_{1}} \\
\omega_{0}=\sqrt[4]{\frac{4 \mathrm{~B}}{5}}=0.710370681 \omega_{1} \\
\mathrm{a}_{2}=\omega_{0}^{2}=0.504626504 \omega_{1}^{2} \\
\mathrm{a}_{1}=\omega_{0} \sqrt{2(1-\mathrm{v})}=0.996506281 \omega_{0}= \\
=0.707888844 \omega_{1}
\end{gathered}
$$

\section{Paszkiewicz wave spectrum}

$$
\begin{gathered}
A=0.812 \cdot 10^{-3}\left(\frac{2 \pi}{T_{1}}\right)^{5}=7.95 \\
B=0.723\left(\frac{2 \pi}{T_{1}}\right)^{4}=1126.8 \\
\omega_{0}=\sqrt[4]{\frac{4 B}{5}}=0.760526133 \omega_{1}
\end{gathered}
$$

$$
\begin{gathered}
\mathrm{a}_{2}=\omega_{0}^{2}=0.5784 \omega_{1}^{2} \\
\mathrm{a}_{1}=\omega_{0} \sqrt{2(1-\mathrm{v})}=0.99650628 \omega_{0}= \\
=0.757869067 \omega_{1} \\
\mathrm{C}=\mathrm{h}_{\mathrm{S}}^{2} \cdot \omega_{1} \cdot 1.9082547 \cdot 10^{-3} \\
\mathrm{a}_{0}=\sqrt{\mathrm{C}}=0.043683574 \cdot \mathrm{h}_{\mathrm{S}} \cdot \sqrt{\omega_{1}}
\end{gathered}
$$

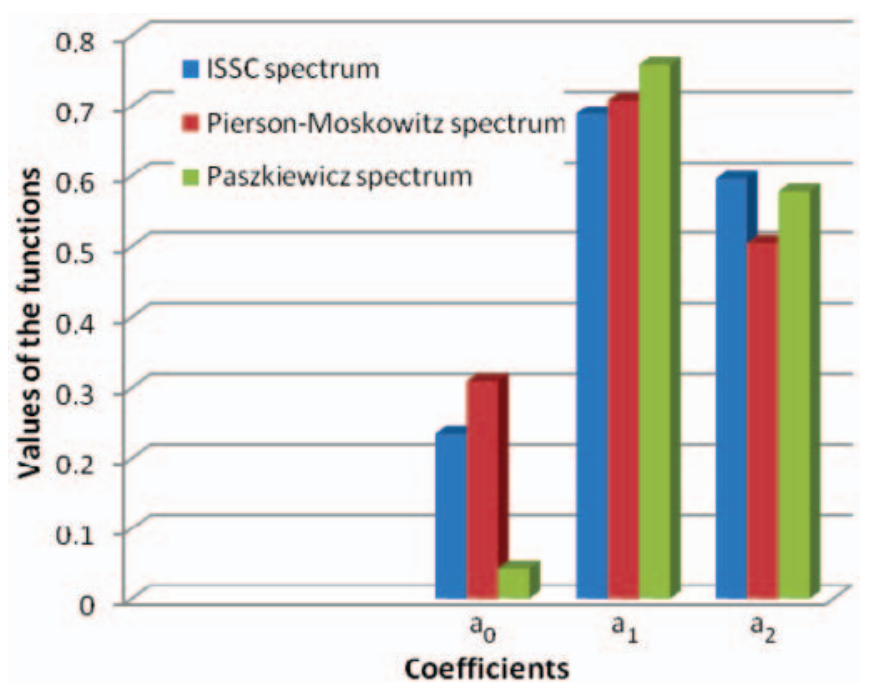

Fig. 2. Distribution of values of the coefficients for the selected wave spectra

Values of the functions for $\omega_{1}=1$ and $h_{s}=1$.

\section{CONCLUSIONS}

1. The coefficients $a_{0}, a_{1}, a_{2}$ which appear in the shape filter (7) were determined. They were determined with taking into consideration the three wave spectra:

a) ISSC (International Ship Structures Congress) spectrum

b) Pierson-Moscowitz spectrum

c) Paszkiewicz spectrum

2. The set of state equations which takes into account symmetrical motions of catamaran, was presented in the form of the matrix equation (given in Tab.2). They constitute the equations of dynamic behaviour of catamaran on irregular wave, called also Itô equations. The quantities $\omega_{0}$, v, C obtained from Eqs. $(18 \div 20)$ made it possible to determine the coefficients $a_{0}, a_{1}, a_{2}$.

3. Further continuation of the subject matter in question is aimed at achieving a solution of the elaborated matrix equation.

Tab. 2. Functional expressions for the coefficients of the selected wave spectra

\begin{tabular}{|c|c|c|c|}
\hline Spectrum of & ISSC & Pierson-Moskowitz & Paszkiewicz \\
\hline $\mathrm{C}$ & $\mathrm{h}_{\mathrm{S}}^{2} \cdot \omega_{1} 0.054985159$ & $\mathrm{~h}_{\mathrm{S}}^{2} \cdot \omega_{1} \cdot 0.095826987$ & $\mathrm{~h}_{\mathrm{S}}^{2} \cdot \omega_{1} \cdot 1.9082547 \cdot 10^{-3}$ \\
\hline $\mathrm{a}_{0}$ & $0.234489144 \mathrm{~h}_{\mathrm{S}} \sqrt{\omega_{1}}$ & $0.309559344 \cdot \mathrm{h}_{\mathrm{S}} \cdot \sqrt{\omega_{1}}$ & $0.043683574 \cdot \mathrm{h}_{\mathrm{S}} \cdot \sqrt{\omega_{1}}$ \\
\hline $\mathrm{a}_{1}$ & $0.688575087 \omega_{1}$ & $0.707888844 \omega_{1}$ & $0.757869067 \omega_{1}$ \\
\hline $\mathrm{a}_{2}$ & $0.596 \omega_{1}^{2}$ & $0.504626504 \omega_{1}^{2}$ & $0.5784 \omega_{1}^{2}$ \\
\hline
\end{tabular}




\section{BIBLIOGRAPHY}

1. Druet Cz.: Sea dynamics (in Polish). Wyd. Uniwersytetu Gdańskiego (Publ. of Gdańsk University), Gdańsk, 2000

2. Kang D., Hasegawa K.: Prediction method of hydrodynamic forces acting on the hull of a blunt-body ship in the even keel condition. Journal of Marine Science and Technology, vol. 12, no. 1 , pp. $1 \div 14,2007$

3. Królicka A.: Multihull vessel excitations in stochastic formulation, Polish Maritime Research, Gdańsk University of Technology, Faculty of Ocean Engineering \& Ship Technology, no. 4, vol. 15, Gdańsk, 2008

4. Królicka A.: Stochastic approach to movements of a multihull on waves. Vibrations in physical systems, Vol.XXIII, pp. $217 \div 222$, Poznań, 2008

5. Plucińska A., Pluciński E.: Probabilistics, Probability calculus, Mathematical statistics, Stochastic processes (in Polish). Wyd. Naukowo-Techniczne (Scientific Technical Publishing House), Warszawa, 2006
6. Rumianowski A.: Research on dynamic behaviour of selected sea floating objects (in Polish), Wyd. PG (Publishing House of Gdańsk University of Technology), Gdańsk, 2003

7. Sobczyk K.: Stochastic differential equations (in Polish), Warszawa, 1996

8. Sobczyk K., Spencer Jr.,B.F.: Stochastic models of fatigue of materials (in Polish). Wyd.Naukowo-Techniczne (Scientific Technical Publishing House), Warszawa, 1996.

\section{CONTACT WITH THE AUTHOR}

Agnieszka Królicka, Ms. C.

Faculty of Ocean Engineering and Ship Technology,

Gdańsk University of Technology Narutowicza 11/12

80-952 Gdańsk, POLAND e-mail:krag@pg.gda.pl phone: (58) 347-11-46 